\title{
Éducation et société
}

Le cas de l'Algérie

Education and society. The case of Algeria

Educación y sociedad. El caso de Argelia

\section{Ahmed Djebbar}

\section{(2) OpenEdition}

1 Journals

Édition électronique

URL : http://journals.openedition.org/ries/2605

DOI : 10.4000/ries.2605

ISSN : 2261-4265

Éditeur

Centre international d'études pédagogiques

Édition imprimée

Date de publication : 1 décembre 1999

Pagination : 45-54

ISSN : $1254-4590$

\section{Référence électronique}

Ahmed Djebbar, «Éducation et société », Revue internationale d'éducation de Sèvres [En ligne], 24 | 1999, mis en ligne le 23 novembre 2012, consulté le 01 mai 2019. URL : http://journals.openedition.org/ ries/2605; DOI : 10.4000/ries.2605 


\title{
Éducation et société
}

\section{Le cas de l'Algérie}

\author{
Ahmed Djebbar \\ Professeur à l'université d'Orsay (Paris XI), \\ ancien ministre de l'Education nationale \\ et de la recherche scientifique de la République algérienne
}

L'école algérienne de ces quarante dernières années est un véritable miroir reflétant les profondes mutations qu'a connues le pays depuis son indépendance. Sa composition humaine, son fonctionnement et le contenu de son projet éducatif sont également des clés pour comprendre les problèmes liés au développement de la citoyenneté dans une société dépourvue de tradition dans ce domaine.

L'Algérie a connu, en moins de cent cinquante ans, une colonisation de peuplement, une guerre de libération nationale puis, après l'indépendance, une gestion du pays par un parti unique. A tout cela, et pour mieux cerner les entraves de toutes sortes qui empêchent le développement de la citoyenneté, il faut ajouter les effets de la crise multiforme (sociale, politique, identitaire) qui a éclaté au grand jour il y a une dizaine d'années maintenant et qui, depuis cette date, la travaille en profondeur. Sur le plan économique, le pays a tenté depuis dix ans, avec peu de moyens et peu d'empressement à vrai dire, de s'adapter aux règles de l'économie de marché, dans une mondialisation trop rapide où il ne trouve pas encore ses marques. Les signes les plus frappants de cette réorientation sans transition sont ceux d'une profonde crise économique qui perdure.

Dans ce double contexte, l'expérience algérienne dans le domaine de l'éducation est très instructive lorsqu'il s'agit d'évaluer le décalage entre la vision qui a nourri la première réforme (toujours à l'œuvre) et les réalités de cette fin de siècle. Elle est surtout révélatrice du fossé existant entre les réussites sur le plan quantitatif et les échecs sur le plan qualitatif, en particulier au niveau de la "fabrication » d'un profil de citoyen. Elle devrait être même un objet d'étude, comme "modèle » dans la mesure où cette expérience a été le fleuron d'une société en pleine expansion et, dans le même temps, un facteur non négligeable dans la maturation de la crise, dans son éclatement et dans ses différentes manifestations : marginalisation, par le chômage, de centaines de milliers de jeunes (souvent victimes de la déperdition scolaire et, parfois, diplômés et sans emploi), déclin des valeurs liées au travail et au savoir, défiance vis-à-vis de l'État et, surtout, violence physique à un niveau jamais atteint auparavant. 


\section{Des orientations généreuses et modernistes...dans un contexte historique singulier}

Il faut, en effet, rappeler que les grandes orientations de l'école algérienne durant la première décennie de l'indépendance étaient une réponse aux pressions très fortes de la population et elles concrétisaient les aspirations de tous ceux qui avaient participé à la lutte pour l'indépendance. Les premières options dans ce domaine ont correspondu à une vision moderniste devant favoriser l'émergence de la citoyenneté, sauf que l'application de ces options s'est faite dans un environnement qui agissait puissamment, et en sens contraire, sur l'institution scolaire.

Un exemple significatif illustre bien ce fait. Il s'agit de la situation très contrastée des femmes dans la société algérienne et du rôle qu'a pu jouer l'école, objectivement, dans la situation que nous observons aujourd'hui. Ce rôle ne peut s'apprécier bien sûr que par rapport à la situation de départ caractérisée par un ensemble d'éléments sociaux, économiques et culturels. Sur le plan social, il s'agissait d'un enfermement (même si la plupart des femmes étaient conditionnées pour le vivre) dans un monde aux frontières invisibles mais réelles et avec ses rapports découlant d'un statut patriarcal ancestral que les bouleversements politiques dans le pays n'ont perturbé qu'à la marge, pourrait-on dire. Sur le plan économique, pour leur grande majorité, les femmes vivaient une situation d'assistées qui perpétuait et renforçait la dépendance vis-à-vis des hommes, de la cellule familiale et de la société au sens large ${ }^{1}$. Sur le plan culturel, la situation de la majorité des femmes dans les années soixante se caractérisait par deux phénomènes : un analphabétisme quasi général et une sous-culture faite de croyances et de pratiques puisées à la fois dans ce qui s'est décanté de douze siècles d'Islam maghrébin et de trois mille ans, au moins, de paganisme berbère.

Dans ce contexte, la fonction citoyenne de l'école a été, dans une première étape, d'ouvrir ses portes aux filles, sans aucune restriction, ni dans le nombre, ni dans la répartition géographique des établissements, ni dans le fonctionnement interne de ces derniers (en particulier au niveau de la mixité qui a été instituée dès le départ). On est ainsi passé de 283000 filles scolarisées en 1962 à plus de 3,5 millions en $1999^{2}$. Il faut d'ailleurs remarquer que même si, effectivement, la scolarisation des filles n'est pas totale, son pourcentage par rapport à celle des garçons n'a jamais cessé d'augmenter lui aussi et, depuis 1998, on obser-

\footnotetext{
1 Parmi les rares secteurs qui employaient les femmes à la veille de l'indépendance et dans les premières années de l'indépendance, il y avait les conserveries de poisson du littoral et l'agriculture (cueillette des agrumes au nord du pays et arrachage de l'alfa dans les steppes du sud).

2 N. Benghabrit-Remaoun, "L'école algérienne : transformations et effets sociaux ", Réflexions, l'école en débat en Algérie, ouvrage collectif, Alger, Casbah Editions, mars 1998, pp. 9-30.
} 
ve même un renversement de tendance dans l'enseignement secondaire puisque le nombre de filles scolarisées est, désormais, plus grand que celui des garçons ${ }^{3}$.

Dans une seconde étape, c'est-à-dire dans les années soixante-dix, et sous l'effet conjugué des revendications des parents eux-mêmes et d'une anticipation des décideurs de l'époque, l'école s'engage dans une véritable opération de promotion sociale en ouvrant plus largement ses portes aux femmes pour des postes d'enseignement, de gestion ou d'encadrement des établissements scolaires. Leur nombre est ainsi passé de moins de 5000 en 1962 à près de 150000 en 1999, soit la moitié du nombre total des enseignants et du personnel de gestion du secteur éducatif ${ }^{4}$.

Mais avant d'aller plus loin, il est peut-être utile de préciser comment a lentement émergé la question de la citoyenneté en Algérie, compte tenu de l'histoire récente du pays, de ses spécificités, mais aussi de ses ambitions et des possibilités de transformation de la société.

Les trois siècles d'administration turque (1516-1830) n'ont introduit aucune modification dans les structures sociales et dans les rapports qui en découlent : l'allégeance tribale était primordiale et les relations avec le pouvoir central se réduisaient souvent à l'acquittement de l'impôt. Avec la colonisation (1830-1962), s'installe dans le pays une nouvelle dynamique qui introduit dans la société autochtone une véritable dichotomie : alors que, dans l'Algérie profonde, on voit se consolider la forme ancienne de l'allégeance, dans les zones de peuplement et de valorisation économique, on assiste à la lente émergence d'une certaine forme de citoyenneté. Elle se manifestera d'abord dans les rapports individuels qu'auront les Algériens avec le nouvel État, à travers son administration locale, puis dans les attitudes et les engagements, toujours individuels, qui vont naître dans le cadre du mouvement national. C'est également pendant cette courte période que commence à s'exprimer une certaine forme de revendication à base linguistique qui donnera plus tard le berbérisme dans sa double expression, politique et culturelle.

La fin de la période coloniale (1927-1962) voit se développer un mouvement de contestation multiforme revendiquant, selon l'appartenance sociale, soit l'intégration, soit l'autonomie, soit l'indépendance pure et simple. Ce mouvement politique, né dans les villes algériennes et au sein de l'émigration en France, ne touchera que partiellement le pays profond qui restera, un certain temps encore, cantonné dans les limites des structures traditionnelles. C'est la décantation interne du courant nationaliste et sa radicalisation par la lutte armée

\footnotetext{
3 Ministère de l'éducation nationale, Rapport d'évaluation de l'activité du ministère de l'Éducation nationale, année scolaire 1997-98, p. 2.

4 Conseil supérieur de l'éducation, Principes généraux de la nouvelle politique éducative et la réforme de l'enseignement fondamental, Alger, mars 1998, partie arabe, annexe, tableau $n^{\circ} 5$.
} 
(1954-1962) qui va en quelque sorte impliquer toutes les couches de la société dans le projet visant à l'indépendance. Sur le plan de la citoyenneté, cette implication a objectivement contribué à l'affaiblissement de l'allégeance tribale et du clivage régionaliste à base linguistique. En effet, ces différents comportements ont eu tendance à se fondre désormais, à des degrés divers bien sûr, dans une attitude, une culture, une mentalité et un discours dont la seule référence était le nationalisme. De plus, par sa «non-fermeture idéologique», ce dernier rendait possibles des initiatives modernistes dans le contexte nouveau d'un pays indépendant et d'une société sans référence précise et contraignante à un modèle de gestion.

\section{Le parti unique instrumentalise l'école...}

C'est cette situation qui va prévaloir au lendemain de l'indépendance et c'est dans ce contexte que vont se dessiner les contours du système éducatif algérien, c'est-à-dire un contexte de nationalisme ouvert dans la mesure où aucune idéologie n'y était privilégiée et ne s'était encore imposée.

Mais, c'est à partir du moment où le système du parti unique a été consacré (avec le choix d'un discours culturel emprunté au courant musulman réformiste et centré sur le concept de la triple appartenance) ${ }^{5}$, que l'instrumentalisation de l'école en vue de la consolidation de ce système est devenue une nécessité stratégique. Cela s'est fait parallèlement à la normalisation de la vie politique puisque certaines préoccupations citoyennes, qui étaient déjà en filigrane dans le projet nationaliste, et d'autres qui avaient été suscitées par l'avènement de l'indépendance, vont être progressivement marginalisées puis combattues au nom du "socialisme spécifique ». Cela a favorisé l'apparition de trois attitudes : la première est l'allégeance au parti unique qui allait générer à la fois un comportement passif et mimétique et un clientélisme forcené, presque à l'image des allégeances traditionnelles. La deuxième est l'indifférence totale par rapport aux problèmes de la cité, avec ce que cela comporte comme engagement citoyen. Cette indifférence va évoluer lentement vers une défiance de plus en plus grande vis-à-vis de l'État et un renoncement aux droits fondamentaux consacrés par la Constitution, comme le droit de vote. La troisième attitude, plus volontariste, mais minoritaire, a été l'engagement dans une opposition active et souvent clandestine puisque toute expression politique et culturelle en dehors du parti unique était réprouvée et souvent combattue.

Dans cet environnement normalisé, l'école, tout en gardant sa vocation de lieu d'apprentissage (qui répondait aux aspirations profondes des parents),

5 C'est-à-dire la nation, la langue arabe et l'Islam. 
allait devenir un enjeu politique, à travers le projet d'arabisation de ses différents paliers. En effet, très vite, les aspects culturels et pédagogiques du projet sont apparus tout à fait secondaires par rapport aux choix politiques. Mais, plus tard, ils sont devenus des instruments pour la pérennisation de ces choix politiques et, par voie de conséquence, des garants d'une certaine orthodoxie dans les conceptions éducatives ${ }^{6}$.

Au niveau des programmes, les orientations nouvelles se sont concrétisées d'abord par la disparition de l'instruction civique à laquelle s'est substituée l'éducation religieuse, créant ainsi les conditions tout à fait légales d'un endoctrinement sans précédent de millions de futurs citoyens. Dans les faits, les programmes officiels de cette matière (qui devaient être centrés sur l'éducation morale à base religieuse) ont été progressivement remplacés, chez beaucoup d'enseignants, par des cours développant la vision du monde et les conceptions politiques des différentes mouvances islamistes qui étaient représentées dans le corps enseignant ${ }^{\text {? }}$.

Au niveau de la langue nationale, on a occulté délibérément tout le patrimoine arabe de la période classique (IX - XIVe siècles), et celui de la période moderne (XIXe - XXe siècles), au profit d'un fond national souvent sans grande envergure. Les chefs-d'œuvre de la littérature internationale (dont les traductions arabes existent pourtant) ont bien évidemment subi le même sort. C'est donc une langue culturellement pauvre mais fortement idéologisée qui s'est finalement imposée.

En histoire, on a mis l'accent, essentiellement, sur le passé récent de l'Algérie en relativisant les grands moments de l'Histoire universelle et même en tronquant l'histoire du Maghreb de certaines périodes cruciales, comme celles qui ont précédé l'avènement de l'Islam. Ce qui a eu pour effet non seulement d'appauvrir la personnalité du futur citoyen en atrophiant sa mémoire mais également de le familiariser avec certaines conceptions de l'histoire à la fois manichéennes et très rétrogrades. La situation était même plus grave pour les élèves de la filière scientifique qui n'étaient pas encouragés à s'intéresser à l'histoire puisque la matière avait été supprimée de l'examen du baccalauréat (comme le français d'ailleurs) ${ }^{8}$.

L'enseignement des sciences n'était pas mieux loti dans la mesure où il était délesté de ses activités d'éveil (qui favorisent le questionnement) et, surtout, de sa dimension culturelle (qui donne un aperçu sur l'élaboration et la cir-

6 A. Djebbar, "Science, culture et patrimoine en Algérie ", Alliage, Nice, n²4-25, 1995, pp. 252-260.

$7 \mathrm{~L}$ 'introduction de linstruction religieuse dans les programmes scolaires n'a pas été une réponse à une quelconque demande ou à un besoin exprimés par les parents. A contrario, l'absence de cet enseignement dans les écoles primaires avant l'indépendance n'a aucunement influé sur la pratique religieuse des Algériens.

8 Ces deux matières ont été réintroduites au baccalauréat en 1993. 
culation des idées scientifiques) ainsi que de sa dimension humaine (qui informe sur les contributions des différents peuples dans les progrès des sciences et des techniques) ${ }^{9}$.

Dans les années soixante-dix, l'école jouait encore pleinement son rôle d'instrument de promotion sociale. Ce qui entretenait chez les parents une forte revendication de scolarisation de leurs enfants. Pour répondre à cette demande, des dizaines de milliers d'enseignants ont été recrutés dans l'urgence. Leur niveau très bas, le contenu de leur formation, parfois sans aucun lien avec la mission qui les attendait et, surtout, le profil idéologique d'un bon nombre d'entre eux (qui avaient été recrutés à l'étranger), vont aggraver la situation en introduisant, ici ou là, des discours idéologiques ou même politiques qui sortaient du cadre imposé par le pouvoir lui-même. C'est ainsi que les quelques valeurs citoyennes qui résistaient encore (comme celles qui accompagnaient la référence à la République et à la nation) ont été relativisées puis dévalorisées par l'évocation constante d'un modèle arabo-islamique très dogmatique. Cette évocation se faisait naturellement à la faveur de l'instruction religieuse mais elle n'a pas tardé à sortir du cadre de cette matière pour investir des enseignements qui en étaient, en principe, très éloignés, comme celui des sciences naturelles ou même des mathématiques.

Occupés par les luttes politiques internes, les décideurs des années quatre-vingt ont laissé se développer, en vase clos, un double phénomène qui résultait à la fois de l'instrumentation initiale du système éducatif (à travers la politique d'arabisation), du profil d'une partie du corps enseignant et de l'activisme d'une minorité d'entre eux (en prise directe avec les mouvements intégristes).

Le premier phénomène a été un abaissement progressif du niveau de l'enseignement qui était visible à travers un certain nombre d'indicateurs : les résultats du baccalauréat (dont la moyenne des taux de réussite n'a jamais atteint les $30 \%$ au cours des deux dernières décennies), la nature et l'étendue des connaissances des diplômés; leur niveau culturel, etc.

Le second phénomène a été l'imprégnation lente de tout le système scolaire par l'islamisme politique et par son idéologie entraînant des comportements et des initiatives contraires à une conception républicaine de l'école et de ses missions.

Il y eut d'abord la non-application de certaines règles de fonctionnement de l'école parce qu'elles ont été considérées comme allant à l'encontre des mœurs d'une bonne société musulmane. C'est le cas de la mixité, de la pratique du sport et de la tenue vestimentaire. Dans ces trois domaines, on a assisté à une

9 A. Djebbar, "Les scientifiques arabes face à leur patrimoine ", Maghreb-Machrek, Paris, Documentation française, $n^{\circ} 105$ (juillet-août-septembre 1984), pp. 48-64. 
bataille feutrée, dans le champ clos de l'école, pour séparer les filles et les garçons, interdire l'éducation physique aux élèves de sexe féminin, obliger les adolescentes et les enseignantes à se couvrir la tête et à porter des habits longs. Cette bataille a révélé de fortes résistances venant plus d'une partie du corps enseignant que des parents d'élèves. Mais ces résistances n'ont pas permis d'endiguer le phénomène, à la fois par manque de culture citoyenne dans la société en général et parmi la majorité des familles, mais également par démission de l'État et du pouvoir qui se sont désintéressés de ces combats jugés secondaires.

Il y eut enfin le dénigrement des valeurs nationales qui sont sensées donner un minimum de cohésion à la société. Parmi ces valeurs, celles liées à la révolution de novembre 1954, qui a mené à l'indépendance du pays, ont été soit occultées, soit minimisées, soit noyées dans les valeurs de la "guerre sainte " revue et corrigée pour qu'elle soit adaptée à la lutte contre d'autres musulmans assimilés à des infidèles. Comme conséquence de ces initiatives, il y eut même le rejet par certains enseignants (et parfois même par certains responsables du secteur éducatif) des symboles de la République (hymne, drapeau, etc.).

Les conséquences de cet endoctrinement à huis clos et dans la longue durée (puisqu'il était possible à un enseignant de suivre une classe pendant six ans) n'ont pas besoin d'une analyse fine pour être dégagées. Elles sont clairement lisibles dans le comportement des élèves encore scolarisés et se retrouvent chez les adolescents qui sont passés par l'école.

A l'école, il y a la banalisation et parfois même l'encouragement de certains comportements, comme la dénonciation, par les élèves, de leurs propres parents pour non-observance de règles religieuses (prière, jeûne, interdiction des boissons alcoolisées). Il y a même parfois, ici ou là, la diffusion d'idéologies racistes et xénophobes, à travers des écrits ou des chansons, sans que cela provoque systématiquement des réactions de rejet et de condamnation dans la société. Chez une bonne partie des adolescents, qu'ils soient diplômés ou qu'ils aient été victimes de la déperdition scolaire, on a observé une absence totale d'esprit critique, d'esprit d'entreprise et de curiosité. On a également remarqué un grand désintérêt pour les problèmes de la cité et le développement d'attitudes dogmatiques à propos de questions scientifiques et culturelles ou de problèmes de société.

Parmi les facteurs qui ont fait que l'école algérienne, dans le contexte bien déterminé que nous avons brièvement évoqué, a pu favoriser l'intolérance, la ségrégation entre les sexes, le dogmatisme, il y a ceux qui sont liés à son environnement immédiat, c'est-à-dire aux familles des élèves. Considérée par la majorité de ces familles comme l'instrument de promotion par excellence, l'école a toujours bénéficié d'un jugement globalement favorable de leur part. Il faut dire que cette image de l'école a été confortée, durant les deux premières décennies de l'indépendance par la capacité qu'avaient alors l'État, ses institutions et ses unités de production, à recruter les diplômés que produisaient l'école et 
l'université. Devant ce succès durable, les parents n'ont pas été, dans leur majorité, très regardant au sujet du contenu de l'enseignement qui était prodigué à leurs enfants. A cela, il faut ajouter un élément capital qui concerne directement le problème de la citoyenneté : les dérives qu'a connues le système scolaire à partir de la fin des années soixante-dix ont été constatées et signalées par des éducateurs et des parents mais cela n'a entraîné aucune action d'envergure permettant de combattre le phénomène. Parmi les raisons de cette inaction, il y avait, bien sûr, l'interdiction, par le parti unique, de tout contre-pouvoir qui aurait pu aider à sensibiliser un plus grand nombre de parents d'élèves. Mais il y avait surtout l'absence de culture citoyenne chez la majorité de ces parents qui avaient pris l'habitude de s'en remettre à l'administration et à l'État pour résoudre tous leurs problèmes.

Tout ce qui vient d'être évoqué était déjà en action, à des degrés divers, bien avant l'avènement de la crise qui a éclaté à partir de 1989 et qui se poursuit encore. Il n'est donc pas étonnant qu'il n'y ait pas eu d'amélioration sur ce planlà, d'autant qu'aucune action à long terme n'a été mise en application dans le but de libérer l'école de toute emprise idéologique et de la préserver, dans le futur, de toute tentation d'instrumentalisation politique.

\section{La citoyenneté dénaturée}

Il nous reste à évoquer d'autres éléments concernant l'école et que la crise a mis en lumière, parfois d'une manière brutale. Ces éléments sont d'ailleurs contradictoires et reflètent bien, par eux-mêmes, la complexité du problème. En effet, cette crise a révélé au grand jour le rôle négatif que peut jouer une institution scolaire lorsqu'elle s'écarte de sa vocation première qui est, bien sûr, de transmettre le savoir et le savoir-faire, mais qui est surtout le lieu où se construit une certaine forme de citoyenneté. Mais, dans le même temps, elle a montré comment cette institution, avec tous ses défauts et ses dysfonctionnements a provoqué, tant à l'extérieur de ses murs qu'à l'intérieur, l'apparition d'une attitude citoyenne visant à défendre ce que l'on pourrait appeler " l'école de la République ", qui était menacée dans son existence même.

Considérée par les citoyens comme l'un des rares symboles positifs de l'État et du pouvoir, l'école est devenue, à partir de 1992, une des cibles privilégiées de l'intégrisme islamique. Empêcher son fonctionnement permettait à la fois d'affaiblir l'État, en révélant son incapacité à faire fonctionner l'institution scolaire, et de lui ôter ses éventuels soutiens dans la population. Alors, pour atteindre ce but, toutes les violences ont été pratiquées : destructions d'établissements, assassinats d'enseignants (parfois dans leur classe en présence de leurs élèves), d'inspecteurs, de responsables de l'éducation. Même des élèves ont été tués dans le but de créer un climat de terreur devant entraîner le refus d'aller à l'école et donc la fermeture des établissements d'enseignement. 
Il faut préciser qu'en plus du but purement politique poursuivi par ces actions, il y avait dans le choix des victimes une volonté d'éliminer tout ce qui pouvait représenter, aux yeux de la population, l'esprit critique, l'esprit d'ouverture, le non-conformisme, la liberté de penser - bref tout ce qui s'opposait à l'endoctrinement, au dogmatisme et à toutes les formes de contrainte qu'avait connues la société à l'ombre du parti unique et que porte en lui, à un niveau plus élevé, le projet islamiste.

Dans le même temps, l'institution scolaire s'est révélée sous un autre jour, moins glorieux. Ce sont, en effet, des enseignants du primaire et du secondaire (parfois des universitaires) qui se sont retrouvés, comme dirigeants, idéologues ou chef de groupes terroristes, à la direction d'un mouvement polycéphale mais uni par une même idéologie et par un même but : l'instauration d'un État théocratique. Pour y parvenir, ces enseignants prêchaient le refus de la démocratie (avec tous ses corollaires sur le plan de la citoyenneté) et l'unicité de la pensée, et ils préconisaient le terrorisme comme prolongement de la lutte pour la prise du pouvoir.

Plus grave encore, ce sont des élèves, parfois scolarisés, qui se sont retrouvés impliqués dans des actions terroristes. Ils ont assassiné des enfants, des femmes, des vieillards, parfois des membres de leur propre famille. L'idéologie au nom de laquelle ils ont commis leurs crimes, ils en ont parfois acquis certains éléments de base à l'école, dans le cadre des cours d'instruction religieuse ou à l'occasion d'improvisation de l'enseignant en marge d'un enseignement ordinaire. Même lorsqu'ils n'ont pas eu l'occasion d'écouter ce type de discours, ils ont évolué dans un environnement scolaire où étaient quotidiennement exprimées des affirmations sur l'inégalité homme-femme, sur la supériorité du musulman, sur l'idée de soumission, sur l'intangibilité du dogme, etc. Tout au long des neuf années de scolarité obligatoire, ces affirmations n'auront été contredites, ou tout au moins atténuées, par aucune éducation à la citoyenneté, par aucune lecture ouvrant de nouveaux horizons de pensée ou suscitant des interrogations contradictoires. Fonctionnant comme la seule culture de base acquise par l'adolescent, ces affirmations, ainsi que les références qui sont à l'arrière-plan, ont ainsi joué le rôle de terreau fertile pour les discours idéologiques et politiques qui ont été prononcés, tout au long des années quatre-vingt, dans des mosquées, dans des universités et parfois à l'occasion de colloques (financés par l'État lui-même et relayés par les médias nationaux).

Aujourd'hui, et après de longues années de silence ou de polémiques politiciennes, un consensus semble se dégager sur le diagnostic concernant le contenu et le fonctionnement du système éducatif. En effet, personne en Algérie 
n'ose plus dire ou écrire que l'école se porte bien et qu'elle n'a pas besoin d'être dépolitisée, dé-idéologisée et profondément réformée au niveau des programmes de la pédagogie et du profil des enseignants. Le moment est donc arrivé, à la faveur de ce consensus, d'engager la réflexion, non pas sur les missions citoyennes de l'école algérienne (qu'il est facile de répertorier et de hiérarchiser en fonction des réalités dont nous avons décrit quelques aspects), mais sur les moyens de mettre en application leur contenu. Cette réflexion est urgente et de ses résultats dépendra la réussite de la future réforme du système éducatif algérien. 